\title{
Nasal paraganglioma: A case report and literature review
}

\author{
Lídio Granato', José Donato Próspero', Dino Martini Filho².
}

\author{
1) Professor, Department of Otolaryngology, \\ 2) Associate Professor, Department of Pathological Sciences. \\ Institution: Department of Otorhinolaryngology of the Brotherhood of Santa Casa de Misericordia de São Paulo São Paulo. \\ São Paulo/SP - Brazil. \\ Mailing address: Lídio Granato - Department of Otolaryngology, Faculty of Medical Sciences of Santa Casa de São Paulo - Rua Dr. Cesário Motta Júnior, 112 - $4 ²$ andar \\ - Zip code: 01277-900 - Vila Buarque - São Paulo / SP - Brazil - Telephone/Fax: (+55 11) 222-8405 - E-mail: drlidio@terra.com.br \\ Article received in July 29, 2008. Article accepted in September 24, 2012.
}

\section{SUMMARY}

Introduction: Paragangliomas are neuroendocrine tumors that most commonly originate in the adrenal gland, a type that is called pheochromocytoma; however, 5-10\% of paragangliomas are extra-adrenal and may arise in any area between the neck and pelvic region along the sympathetic nervous system. Those located in the head and neck comprise $3 \%$ of extra-adrenal tumors, with the majority originating in the tympanic-jugular region and carotid body.

Objective: To present a rare case of nasal paraganglioma and review the literature.

Case report: The patient was submitted to medial subtotal maxillectomy, and her clinical findings, diagnostic data, and treatment outcome were recorded.

Conclusion: Paragangliomas are considered benign tumors, but they occasionally display a malignant character. The most important finding in this case was the need for total resection of the tumor to avoid recurrence.

Keywords: Paraganglioma, Neuroendocrine Tumors, Nasal Cavity, Adrenal Gland Neoplasms, Neoplasms

\section{INTRODUCTION}

Paragangliomas are neuroendocrine tumors. Typically, these tumors originate from the adrenal gland, and in such cases, they are named pheochromocytomas. However, $5-10 \%$ of these tumors are extra-adrenal and may appear in any area between the neck and the pelvic region along the sympathetic nervous system $(1,2)$.

These tumors have been described in the orbit, larynx, nasopharynx, thyroid gland, vagus, mediastinum, lung, duodenum, aortic region (organ of Zuckerkandl), retroperitoneal region, and bladder (1).

The term paraganglioma is currently suggested by several authors for all tumors derived from paraganglion tissue regardless of the result of chromaffin dye staining. This is based on the data of Zak and Lawson revealing that less than $1 \%$ of paragangliomas exhibit only endocrine activity (3).

There is only a single case report of ACTH-producing nasal paraganglioma associated with Cushing's syndrome. Due to the uncertainty of chromaffin dye as a predictor of tumor endocrine function, the generic term paraganglioma was selected for these tumors (4).

Paragangliomas located in the head and neck comprise $3 \%$ of extra-adrenal tumors, with the majority originating in the tympanic-jugular region and carotid body (1).

The appearance of these lesions in the nasal cavity and paranasal sinuses is extremely rare. These lesions cause obstruction and nasal bleeding, and they can be confused with other benign and malignant lesions. On occasion, they can be confused with glomus tumors because even though they are immunologically and histologically different, both share the feature of great vascularity (5). While there are doubts regarding the origin of this tumor in the sinonasal cavity, in most cases described in the literature, the regions most affected were the middle turbinate and ethmoid sinus (6).

Owing to the rarity of the clinical presentation, we report the case of a 61-year-old female patient paraganglioma.

\section{CAse Report}

A61-year-old woman complained of nasal obstruction and bleeding for 20 years.

The patient reported that since the first occurrence of the disease, she had been experiencing nasal obstruction and bleeding through the right nostril, having recently been hospitalized for 4 days to curb the bleeding. 
In the ENT examination, we observed a mass in the right nostril that shifted the wall of the maxillary sinus sideways and pushed the nasal septum to the opposite side. The left nostril was entirely blocked. We observed a slight bulging of the palate in its right half.

Contrast-enhanced computed tomography (CT) in the axial position revealed a mass with an expansive character completely occupying the right nasal cavity from the foyer to the nasopharynx. This mass shifted the septum to the opposite side, completely blocking the contralateral nostril. It also shifted the medial wall of the maxillary sinus outward. The use of contrast illustrated the heterogeneous aspect of the lesion with more emphasis on the periphery of the tumor (Figure 1).

The coronal position revealed a large mass occupying the entire nasal cavity, and the nasal septum and medial wall of the maxillary sinus was nearly unidentifiable because of ipsilateral tumor invasion through the middle meatus. The bone of the hard palate was shifted down in their 2/ 3 right side (Figure 2).

Due to the clinical characteristics of the lesion, including its long progression, growth, and expansive nature, it was decided to perform surgery and avoid a biopsy, which could result in significant bleeding based on the patient's history of bleeding.

Subtotal medial maxillectomy was performed using a degloving exposure. After obtaining access to the right ethmoid sinus, the remaining medial bone wall was removed from the right maxillary sinus using the Caldwell-Luc technique with preservation of the inferior meatus mucosa and its inferior turbinate. An incision was made in an anterior-to-posterior direction in the lateral mucosa of the nasal cavity, immediately next to the floor to lift up the mucosal wall, with the objective of achieving a wide sinonasal cavity, which promotes tumor resection by detachment.

The tumor occupied the entire right nasal cavity, extending into the nasopharynx and invading the maxillary sinus meatus. The septum was completely displaced and thinned but without perforation. Both the septum and the medial wall of the maxillary sinus mucous were placed in their original position (Figures 3A and B). There was significant bleeding during surgery, but this did not impair tumor resection.

Two years after surgery, the patient exhibited a good outcome. Although the right nasal cavity remained wide, the mucosa remained in good condition, and the patient had no complaints.

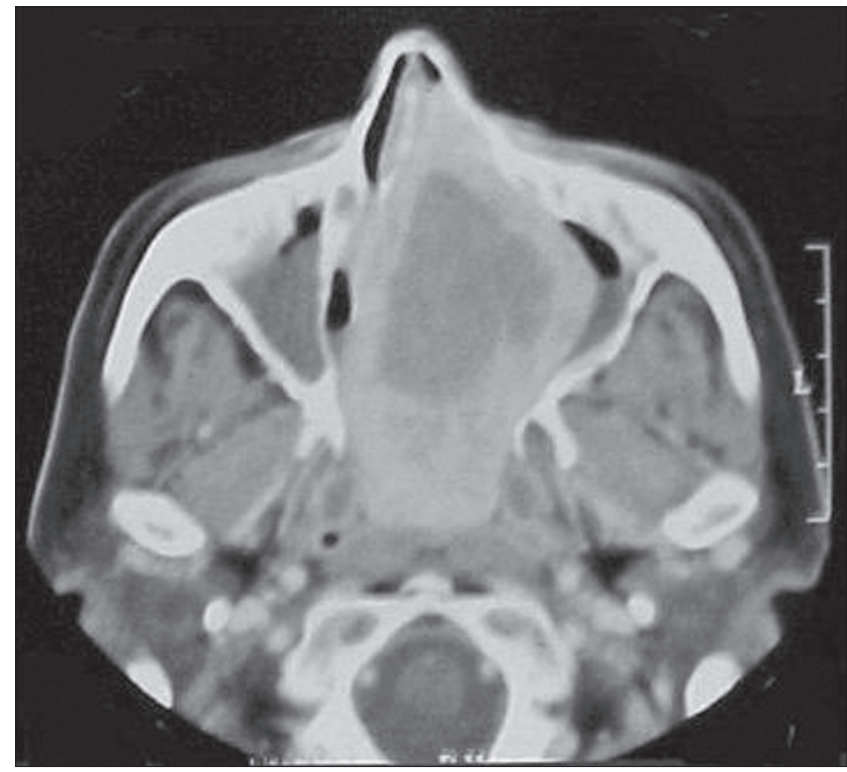

Figure 1. Computed tomography with iodinated contrast in the axial position revealing an expansive and heterogeneous lesion.

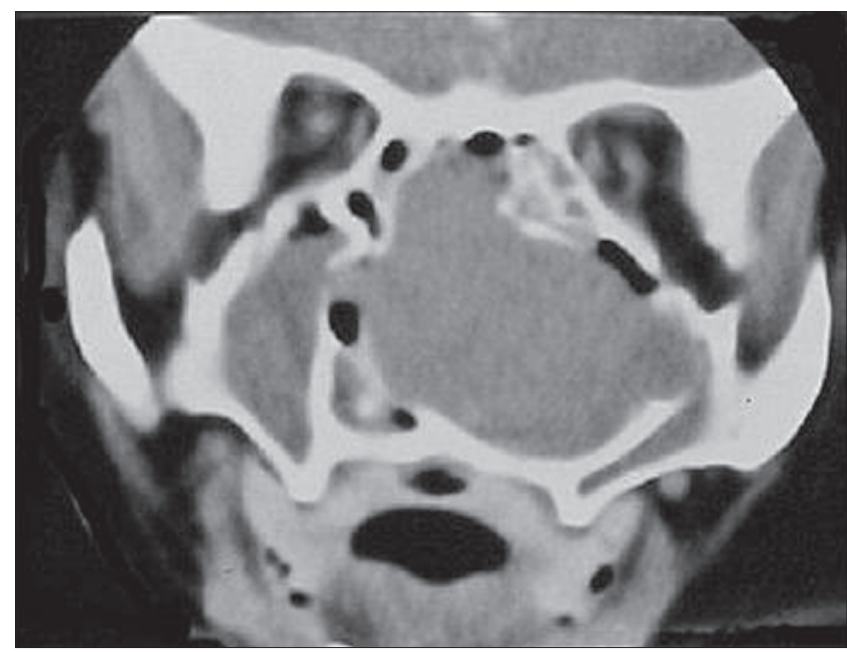

Figure 2. Computed tomography in the coronal position revealing a lesion occupying the entire right nasal cavity with invasion of the maxillary sinus through the middle meatus.

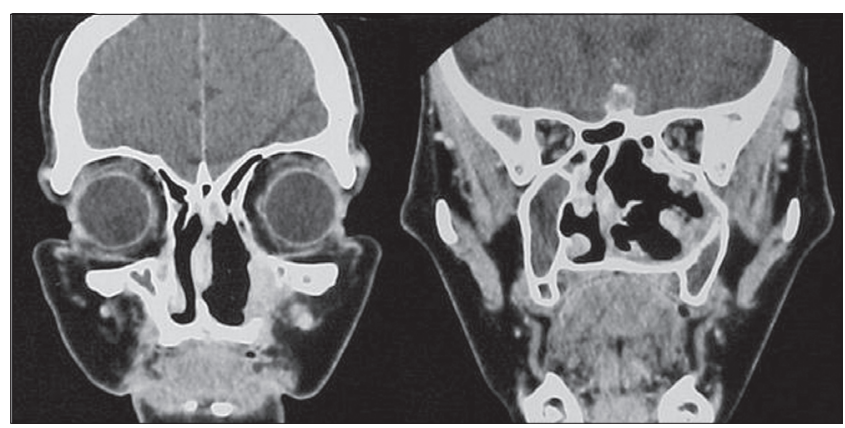

Figure 3. A. Centered septum B. Wide nostril with mucosa in good condition. 


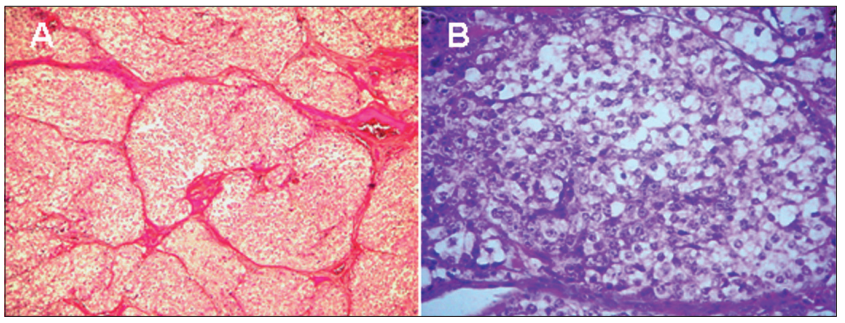

Figure 4.

The pathological examination revealed neoplasm characterized by rounded or polyhedral cells with vesicular nucleus and scarce cytoplasm of imprecise limits. These cells were arranged in cords, and they assumed an alveolar arrangement in blocks delimited by vascularized connective tissue septa (Figures $4 \mathrm{~A}$ and $\mathrm{B}$ ).

The neoplastic cells displayed an affinity for chromic acid, and therefore, they were chromaffin cells.

Diagnosis: Paraganglioma.

\section{DisCUSSION}

Nasal paragangliomas are very rare; until 2002, there were only 27 cases reported in the literature. The tumor occurs in a wide range of patients aged between 8 and 89 years, with middle-aged females being most affected ( 7 ).

The nasal cavity is more affected by the tumor than the sinuses; most paragangliomas originate in the lateral nasal wall and middle turbinate. Regarding the location of this tumor in the nasal cavity, it is worth mentioning that in the first 3 cases described in the literature, the source of the tumor was the nasal septum (8). Tumors originating from the inferior turbinate and the posterior portion of the choana have also been described (9).

The ethmoid sinus is most commonly affected by nasal paragangliomas. Talbot published a case with exclusive delocalization of the maxillary sinus (10).

The origin of paragangliomas in the nasal cavity is still not well understood. Under normal conditions, there is no evidence of noncromaffin tissue in the nasal passages. Glenner and Grimley (2) reported that these tumors may arise from vestigial cell nests originated in fetal development.

Paragangliomas are slow-growing, expansive, multicentric tumors. Approximately 3\% of these tumors are extra-adrenal, and up to $26 \%$ of patients with this tumor have a positive family history, illustrating the existence of genetic predisposition (11).
The clinical presentation is usually characterized by nasal obstruction, repeated and profuse bleeding, a runny nose, facial swelling, and ipsilateral pain.

Generally, a reddish polypoid mass occupying the nasal cavity and migrating to the sinuses is observed (12). In our patient, the mass was attached to the middle turbinate compromising the right ethmoid and maxillary sinuses with bone erosion.

Examinations such as contrast-enhanced CT and magnetic resonance imaging are essential for diagnosis. CT reveals an important impregnation with iodinated contrast, thus illuminating the great vascular component of the tumor. The presence of a well-individualized expansive lesion is suggestive of benign tumors (11).

Arteriography can be used to study the vascular network of the tumor and identify the nutrient artery for possible embolization. This procedure can be excluded in most cases together with biopsy if the surgeon is sure about the nature of the lesion.

The procedure used for our patient was external broad access without embolization.

Macroscopic examination of the paraganglioma revealed firm and apparently encapsulated lesions.

Histological examination revealed a neoplasm with epithelioid cells with round nuclei and eosinophilic cytoplasm, forming nests called "zellballen," separated by a network of capillaries rich in reticulin (13).

A special histological stain is used to identify the neurosecretory cytoplasmic granules of these cells.

The neoplastic cells exhibit affinity for chromic acid (13).

The chosen treatment was surgery. Access can be made via conventional techniques, such as lateral rhinotomy, exposure by degloving to expand the access to the sinonasal region, or endoscopic surgery. Occasionally, depending on the size of the tumor, a mixed endonasal and extranasal approach can be used.

The most important feature of treatment is resection of the tumor, as relapse always occurs when surgery is incomplete. According to Arens et al. (14), the rate of recurrence of this benign tumor is $10 \%$.

The greatest difficulty in the removal of this tumor is bleeding due to its rich vascularity. Embolization has been used to reduce blood volume during surgery (15). 
The incidence of paragangliomas of the head and neck ranges from 4 to $19 \%$ (13), and metastasis may occur in 9\% of cases (5).

Paragangliomas, although considered benign tumors, can present some histological features that suggest more aggressiveness such as cytological pleomorphism, nuclear atypia, high mitotic atypia, foci of necrosis, and vascular invasion. Some of these changes have been observed in benign tumors (13).

Thus far, there is no reliable marker of malignancy for these tumors, and thus recurrence, distant metastasis, and bone invasion are the best parameters to define the malignancy of the tumor (5).

The differential diagnosis can be made with, for example, hemangiopericytoma, olfactory neuroblastoma, and angiosarcoma, among others (7).

Some authors have used radiotherapy occasionally, but it should be reserved for special cases and as an adjunctive therapy (4). Chemotherapy is ineffective for the treatment of paraganglioma (13).

\section{CONCLUSION}

The appearance of these lesions in the nasal cavity and paranasal sinuses is extremely rare. Clinically, they cause obstruction and nasal bleeding, and they can be mistaken for other benign and malignant lesions. They are histologically and immunologically different from glomus tumors, but these lesions should not be confused with those even though they share the characteristic of great vascularity.

The treatment should be surgical, as other therapies are palliative and surgery tends to be radical in terms of complete elimination and prevention of recurrence.

\section{ACKNOWLEDGMENTS}

We thank the Support Center for Publication of the Faculty of Medical Sciences of Santa Casa de São Paulo NAP-SC for technical and scientific support of the publication of this manuscript.

\section{REFERENCES}

1. Fries JG, Chamberlin JA. Extra-adrenal pheochromocytoma: literature review and report of a cervical pheochromocytoma. Surgery. 1968;63:268-79.
2. Glenner GG, Grimley PM. Tumors of the extra-adrenal paraganglion system (including chemorececptors): Atlas of tumor pathology, $2^{\text {nd }}$ ed. Washington, DC.: Armed Forces Institute of Pathology, 1974.

3. Zak FG, Lawson W. The paraganglionic chemoreceptor system. Springer, New York: Springer-Verlag, 1982.

4. Apple D, Kreines K. Cushing's syndrome due to ectopic ACTH production by a nasal paraganglioma. Am J Med Sci. $1982 ; 283: 32-5$.

5. Battiata AP, Vander Ark W, Adair C, Mair E. Pathology forum: quiz case. Diagnosis: intranasal glomus tumor. Arch Otolaryngol Head Neck Surg. 2001;127:329-30.

6. Watson DJ. Nasal paraganglioma. J Laryngol Otol. 1988;102:526-9.

7. Ketabchi S, Massi D, Santoro R, Franchi A. Paraganglioma of the nasal cavity: a case report. Eur Arch Otorhinolaryngol. 2003;260:336-40.

8. Potter AJ Jr, Khatib G, Peppard SB. Intranasal glomus tumor. Arch Otolaryngol. 1984;110:755-6.

9. DeBord BA. Unusual presentation in otolaryngology. Surg Clin North Am. 1972;52:472-83.

10.TalbotAR. Paraganglioma of the maxillary sinus. JLaryngol Otol. 1990;104:248-51.

11. Mavez M, Rusty C. In Bailey B, Healy G, Johnson J. Head \& Neck Surgery-Otolaryngology, 3nd ed. Philadelphia: Lippincott Williams \& Wilkins, 2001.

12. Fu YS, Perzin KH. Nonepithelial tumors of the nasal cavity, paranasal sinuses and nasopharynx: a clinicopathologic study: I. General features and vascular tumors. Cancer. 1974;33:1275-88.

13. Nguyen QA, Gibbs PM, Rice DH. Malignant nasal paraganglioma: a case report and review of the literature. Otolaryngol Head Neck Surg. 1995;113:157-61.

14. Arens C, Dreyer T, Eistert B, Glanz H. Glomangioma of the nasal cavity. Case report and literature review. ORL J Otorhinolaryngol Relat Spec. 1997;59:179-81.

15. Schuller DE, Lucas JG. Nasopharyngeal paraganglioma. Report of a case and review of literature. Arch Otolaryngol. 1982;108:667-70. 